\title{
Comparison of the quality of life among persons with lung cancer, before and after the chemotherapy treatment ${ }^{1}$
}

\author{
Priscila Isolani de Oliveira² \\ Carlos Alberto de Castro Pereira ${ }^{3}$ \\ Angélica Gonçalves Silva Belasco ${ }^{4}$ \\ Ana Rita de Cássia Bettencourt ${ }^{4}$
}

Objective: this prospective study aimed to assess the quality of life related to health (QLRH) of patients with lung cancer after chemotherapy treatment. Method: The QLRH was assessed using the questionnaires Quality-of-Life Questionnaire-Core 30 (QLQ-C30) and Lung Cancer Module (LC13), version 3.0. Results: the sample was made up of 11 women and 19 men, with an average age of 68 years (51-87 years). After the chemotherapy treatment, the authors observed a clinically-relevant improvement in general quality of life, as well as in the symptoms of dyspnea, insomnia, hemoptysis, cough, thoracic pain, pain in the arm/shoulder, and financial difficulty. There was a worsening on the functional scale which assesses role performance and symptoms of fatigue, nausea and vomiting, sensory neuropathy, pain in other parts, constipation, loss of appetite and alopecia. Conclusion: although the patients have an improvement of their QLRH and symptoms related to the lung cancer after the chemotherapy treatment, there was a worsening of the symptoms which resulted from the toxicity of the chemotherapy medications.

Descriptors: Quality of Life; Carcinoma, Bronchogenic; Drug Therapy.

\footnotetext{
${ }_{1}$ Paper extracted from Master's Thesis "Avaliação prospectiva da qualidade de vida versus resposta tumoral em pacientes com câncer de pulmão" presented to Escola Paulista de Medicina, Universidade Federal de São Paulo, São Paulo, SP, Brazil.

2 RN, Specialist in Clinical and Surgical Nursing, Hospital Servidor Público Estadual, São Paulo, SP, Brazil.

${ }^{3}$ PhD, Physician, Departamento de Pneumologia, Universidade Federal de São Paulo, São Paulo, SP, Brazil.

${ }^{4}$ PhD, Adjunct Professor, Escola Paulista de Enfermagem, Universidade Federal de São Paulo, São Paulo, SP, Brazil.
}

Corresponding Author:

Priscila Isolani de Oliveira

Universidade Federal de São Paulo. Escola Paulista de Enfermagem

Rua Napoleão de Barros, 754

Vila Mariana

CEP: 04024-002, São Paulo, SP, Brasil

E-mail: priscilaisolani@hotmail.com 


\section{Introduction}

Worldwide, lung cancer is the most common type of cancer. In Brazil, it has become one of the most important public health problems, and is among the cancers which cause the greatest mortality among men. It is estimated that in 2012, there were 17,210 new cases of lung cancer among men, and 10,110 among women. This type of cancer is considered an aggressive illness and is generally detected in the advanced stages, as the symptoms in the initial stages of the disease are not common. As a result, lung cancer remains a highly lethal disease, in which over $86 \%$ of the patients die in the first five years following diagnosis. Lung cancer does not present as a disease with uniform behavior, as it has different histological types with different biological activities and aggressiveness, with non-small-cell lung carcinoma being the most frequent type of lung cancer(1-2).

The value of chemotherapy in the treatment of advanced non-small cell lung cancer has been proven over the last decade, there being an increase in survival in comparison with palliative care. The benefit, however, is modest, as chemotherapy's impact on quality of life remains an unknown ${ }^{(3-4)}$.

All this effort from science and technology in prolonging life is significant, but cannot be viewed in isolation. It is fundamental that the prolonging of survival should be associated with better quality of life $(Q L)^{(5)}$. Quality of life related to health (QLRH) is "the value assigned to duration of life as modified by the impairments, functional states, perceptions, and social opportunities that are influenced by disease, injury, treatment, or policy ${ }^{(6) " .}$

Due to the large number of symptoms and morbidities caused by lung cancer, the assessment of QLRH has become an essential component in the management of cancer, and its evaluation must be incorporated as a matter of routine, helping in the comparison of different therapeutic regimes, and consequently in the choice of the most appropriate mode ${ }^{(7)}$.

In Brazil, there have been few scientific works discussing the quality of life of patients with lung cancer undergoing chemotherapy treatment. By far the majority of studies have been undertaken in other countries, principally in developed countries which, due to the cultural and socio-economic differences, do not reflect the context of Brazilian patients. The present study's objective was to assess the changes in the QLRH of patients with lung cancer before and after chemotherapy treatment.

\section{Methods}

This is an observational and cross-sectional study, carried out in a public hospital in São Paulo, between June 2007 and February 2009.

The study included a convenience sample made up of 30 patients who met the following inclusion criteria: age over 18 years old, a diagnosis of non-small cell lung cancer confirmed by pathology, staging III-B or IV, indicated for treatment with chemotherapy exclusively, a Karnofsky Index of over 50 including patients without complaints, with moderate symptoms, or who need occasional care, but who are still capable of meeting the majority of their needs. Patients were excluded who had a Karnofsky Index below 50 - as these require considerable care and frequent medical care, those with a prior diagnosis of cancer, and the patients who refused to participate in any phase of the study. The research was approved by the Research Ethics Committee and all the patients signed the terms of Free and Informed Consent.

The instruments included in this study included a socio-demographic and clinical form, the Karnofsky functional performance scale, and the QLRH questionnaires from the European Organization for Research and Treatment of Cancer (EORTC), these being the Quality of Life questionnaire-core 30 (QLQ-C30) and the Lung Cancer Module (LC-13), version 3.0(8-9). The QLRH questionnaires were translated and validated for the Portuguese language and their use was authorized by the $\operatorname{EORTC}^{(10-11)}$.

Data collection was carried out in an environment set aside for the purpose, on a date coinciding with the patient's appearance in hospital, prior to either the meeting with the doctor or the procedure. In the first phase of data collection, the Karnofsky scale, the socio-demographic and clinical form, and the QLRH questionnaires were administered to all the patients with suspected lung cancer. After the diagnosis had been confirmed and treatment prescribed, the disease's clinical progression was monitored, and after the third cycle of chemotherapy, the QLRH questionnaires were administered for the second time. The institution's chemotherapy protocol has, on average, six cycles, with all patients being re-assessed after the third cycle of chemotherapy. The assessment of survival was undertaken in February 2009, by checking the hospital's records of deaths. In the case of patients for whom there was no hospital record of death, an active search was made by telephone to verify the progression of the disease. 
For characterization of the sample, the following variables were collected from the socio-demographic and clinical questionnaires: sex, age, level of schooling, marital status, religion, employment situation, whether the person smoked, initial symptoms, histological diagnosis, staging, chemotherapy regime, tumor response and survival.

The Karnofsky Scale comprises a numerical scale (10-100) which assesses the patients' physical limitations, signs and symptoms, need for care and general status. On the scale, the higher the score is, the better the functional performance, and the lower the score, the more debilitated the patient is ${ }^{(8)}$.

The QLQ-C30 is a questionnaire made up of 30 questions distributed in five scales of functionality, representing the domains of the QLRH (physical function, cognitive function, emotional function, social function and role performance); three scales of symptoms (fatigue, pain, nausea and vomiting); six further items assessing symptoms commonly associated with cancer patients (dyspnea, lack of appetite, insomnia, constipation and diarrhea); a scale for global quality of life and health, and a scale for assessing the financial impact of the treatment and of the disease. The QLQ-LC13 is a complementary module of the QLQ-C30, specifically for evaluating quality of life in patients with lung cancer. The questionnaire is made up of 13 questions which assess the symptoms associated with lung cancer, by means of a scale for dyspnea and other items (cough, hemoptysis, dyspnea and pain in a specific place); effects related to the treatment (pain in the throat, dysphagia, sensory neuropathy and alopecia) and treatment of the pain ${ }^{(9)}$.

The values for scoring the scales and the individual items of the QLQ-C30 and LC13 vary from 0 to 100 . In the interpretation of the functional scales and general state of health/QL, the higher the score, the better the functional index and $\mathrm{QL}$, therefore, positive $\Delta=$ worsening of functional level/QL, while negative $\Delta=$ improvement of functional level and QL. For the scales or items which assess the symptoms, the higher the score, the higher the indexes of the symptoms and problems, therefore positive $\Delta=$ improvements in symptoms while negative $\Delta=$ worsening of symptoms ${ }^{(9)}$.

The data was stored and analyzed using the SPSS (Statistical Package for the Social Sciences), program, version 13.0. The descriptive analyses were undertaken to characterize the sample and included the calculation of absolute frequency, percentage, and measurements of central tendency. For the comparative analysis of the QLRH before and after the chemotherapy treatment, the paired t test was used, due to the normal distribution of the data. The Kaplan-Meier method was used for the survival curve.

As a measure of reliability, the Cronbach alpha co-efficient was calculated, to assess the internal consistency of the QLRH assessment questionnaires, in which a value equal or superior to 0.70 was considered adequate to ensure the scale's good reliability. The test showed satisfactory reliability for the scales of global quality of life and health, role performance, emotional function, social function, fatigue and dyspnea. The scales for pain, cognitive function, the scales for physical function and nausea and vomiting had a dissatisfying performance, with values below 0.70 (Table 1). One of the factors which may have led to the low consistency was the number of items on the scale, as the Cronbach alpha is fairly sensitive to the number of items on the scale and to the number of points it has ${ }^{(12)}$.

Table 1 - Test of the reliability of the QLQ-C30 and LC-13 instruments administered to thirty patients with bronchial carcinoma, São Paulo, SP, Brazil, 2012

\begin{tabular}{lcccc}
\hline & Scale & No items & N & Cronbach alpha* $^{*}$ \\
\hline Global Health/QL & QL2 & 2 & 30 & 0.94 \\
Social Function & SF & 2 & 30 & 0.94 \\
Dyspnea & LCDY & 3 & 30 & 0.76 \\
Role Performance & RF2 & 2 & 30 & 0.73 \\
Emotional Function & EF & 4 & 30 & 0.73 \\
Fatigue & FA & 3 & 30 & 0.71 \\
Physical Function & PF2 & 5 & 30 & 0.63 \\
Pain & PA & 2 & 30 & 0.63 \\
Nausea and Vomiting & NV & 2 & 30 & 0.60 \\
Cognitive Function & CF & 2 & 30 & 0.56 \\
\hline
\end{tabular}

* Cronbach alpha test 
For interpretation of the clinically-relevant results, the authors took into account the delta $(\Delta)$ value corresponding to the average score of the QLRH measurements prior to chemotherapy minus the average score after chemotherapy. Delta values varying between 5 and 10 points between the groups were defined as clinically-relevant, while values over 10 points represented a large change in the domain assessed(13-14). The confidence interval used was $95 \%$. For this study, a level of significance below 0.05 was considered statistically-significant.

\section{Results}

In the first phase of the study, 61 patients were assessed, with 31 being excluded subsequently: 23 patients died before completing the second phase of the study; 6 patients refused to participate in the study ( 4 in the first phase and 2 in the second); a further 2 patients did not conclude the second phase of the research because they had chosen to carry out the treatment in another service. The principal characteristics of the 30 patients who concluded the study are shown in Table 2.

Table 2 - Demographic and clinical data of the patients with lung cancer, São Paulo, SP, Brazil, 2012

\begin{tabular}{lc}
\hline \multicolumn{1}{c}{ Characteristics } & $\mathrm{N}=\mathbf{3 0}$ \\
\hline Age $(\text { years })^{*}$ & $68(8.8) / 51-87$ years \\
Sex & \\
Female/ Male & $11(37 \%) / 19(63 \%)$ \\
Race $^{\dagger}$ & \\
White/ Not white $^{\prime}$ & $25(83 \%) / 05(17 \%)$ \\
Smoking & \\
Smokers/ Ex-smokers/ Non-smokers & $12(40 \%) / 13(43 \%) / 05(17 \%)$ \\
Initial symptoms $^{\dagger}$ & \\
Dyspnea / Weight loss / Cough & $17(57 \%) / 11(37 \%) / 23(77 \%)$ \\
Karnofsky* & \\
Pre-chemotherapy / Post-chemotherapy & $76(8) / 72(8)$ \\
Staging & \\
IIIB/IV & $14(47 \%) / 16(53 \%)$ \\
Chemotherapy Protocol & \\
Cisplatin + Gemcitabine & $19(63 \%)$ \\
Gemcitabine + Carboplatin & $6(20 \%)$ \\
Carboplatin + Paclitaxel & $2(07 \%)$ \\
Carboplatin + Pemetrexed & $2(07 \%)$ \\
Paclitaxel + Gemcitabine & $1(03 \%)$ \\
Tumor response & \\
Response/Without response & $16(53 \%) / 14(47 \%)$ \\
\hline
\end{tabular}

* Average, Standard Deviation and Minimum and Maximum Values; †Frequency and Percentages

The Karnofsky Index had a median of 80 points before treatment and a median of 70 points after the treatment. In the assessment of the Karnofsky scale, $6.7 \%$ of the patients progressed with an improvement in the Performance Status, $53.3 \%$ of the patients remained stable, and $40 \%$ presented a statistically-significant worsening ( $z=2.69, p=0.007$, Wilcoxon test).

The results of the assessment of the QLRH domains, evaluated by the QLQ-30 questionnaire, before and after the third cycle of chemotherapy treatment, are presented in Table 3. One may observe that after the third cycle of chemotherapy, there was a clinicallyrelevant improvement in quality of life ( -7.5 points), as well as in the symptoms of dyspnea (15.5 points and $\mathrm{p}=0.037$ ), insomnia (14.4 points), and financial difficulties ( 5.5 points). After chemotherapy treatment, a worsening was observed in the symptoms of fatigue (-5.9 points) and nausea and vomiting (-8.9 points). The symptoms of constipation and loss of appetite were the most intense with variation from -16.7 and -17.8 points. There was a worsening in the functional scale which assesses role performance, with 5.6 points. 
Table 3 - Comparison of Mean Score of the QLQ-C30 Pre- and Post-Chemotherapy Treatment in 30 patients with bronchial carcinoma, São Paulo, SP, Brazil, 2012

\begin{tabular}{|c|c|c|c|c|c|c|c|}
\hline & \multicolumn{2}{|c|}{$\begin{array}{l}\text { Pre-chemotherapy } \\
\qquad \mathrm{N}=30\end{array}$} & \multicolumn{2}{|c|}{$\begin{array}{l}\text { Post-chemotherapy } \\
\qquad \mathrm{N}=30\end{array}$} & \multirow[t]{2}{*}{$\Delta$} & \multirow[t]{2}{*}{$\mathbf{t}^{*}$} & \multirow[t]{2}{*}{ p } \\
\hline & Mean & SD & Mean & SD & & & \\
\hline Dyspnea & 38.8 & 39,2 & 23.3 & 26.4 & $15.5^{\dagger}$ & 2.19 & $0.037^{\ddagger}$ \\
\hline Insomnia & 36.6 & 39,4 & 22.2 & 33.1 & $14.4^{\dagger}$ & 1.65 & 0.108 \\
\hline Role Performance & 70.0 & 27,8 & 64.4 & 37.3 & $5.6^{\dagger}$ & 0.62 & 0.538 \\
\hline Financial Difficulties & 26.6 & 34,3 & 21.1 & 34.4 & $5.5^{\dagger}$ & 0.694 & 0.493 \\
\hline Social Function & 78.3 & 18,6 & 73.8 & 31.1 & 4.5 & 0.75 & 0.455 \\
\hline Physical Function & 72.4 & 19,4 & 68.2 & 16.0 & 4.2 & 1.33 & 0.191 \\
\hline Cognitive Function & 78.8 & 26.9 & 76.6 & 25.7 & 2.2 & 0.55 & 0.580 \\
\hline Pain & 23.8 & 26.1 & 23.3 & 30.1 & 0.5 & 0.079 & 0.937 \\
\hline Emotional Function & 66.9 & 25.3 & 68.6 & 25.9 & -1.7 & -0.320 & 0.751 \\
\hline Diarrhea & 1.1 & 6.0 & 5.5 & 15.3 & -4.4 & -1.43 & 0.161 \\
\hline Fatigue & 30.0 & 27.5 & 35.9 & 30.1 & $-5.9^{\dagger}$ & -0.79 & 0.431 \\
\hline General State of Health/QL & 58.3 & 23.9 & 65.8 & 23.6 & $-7.5^{\dagger}$ & -1.52 & 0.139 \\
\hline Nausea and Vomiting & 7.2 & 15.5 & 16.1 & 24.9 & $-8.9^{\dagger}$ & -1.72 & 0.096 \\
\hline Constipation & 18.8 & 27.2 & 35.5 & 39.0 & $-16.7^{\dagger}$ & -1.94 & 0.062 \\
\hline Loss of Appetite & 23.3 & 36.2 & 41.1 & 41.6 & $-17.8^{\dagger}$ & -1.88 & 0.069 \\
\hline
\end{tabular}

*Paired t test; +Clinically-relevant scoring; $\neq p<0.05$;

Table 4 presents the data from the average scores of the specific questionnaire QLQ-L13 before and after the third cycle of chemotherapy treatment. In the assessment of the quality of life related to health, with the lung-cancer-specific questionnaire, there was improvement in the symptoms of hemoptysis (8.8 points and $p=0.043)$, cough (8.9 points), thoracic pain ( 10.0 points) and pain in the arm/shoulder ( 12.3 points) after chemotherapy treatment. In contrast, there was a worsening of alopecia ( -24.5 points and $p=0.000)$, sensory neuropathy ( -8.9 points) and pain in other parts (-5.6 points).

Table 4 - Comparison of Mean Scores for the QLQ-LC13 Pre- and Post-Chemotherapy Treatment in thirty patients with bronchial carcinoma, São Paulo, SP, Brazil, 2012

\begin{tabular}{|c|c|c|c|c|c|c|c|}
\hline & \multirow{2}{*}{\multicolumn{2}{|c|}{$\begin{array}{c}\text { Pre-Chemotherapy } \\
\qquad \mathrm{N}=\mathbf{3 0}\end{array}$}} & \multirow{2}{*}{\multicolumn{2}{|c|}{$\begin{array}{l}\text { Post-Chemotherapy } \\
\qquad \mathrm{N}=30\end{array}$}} & \multirow{3}{*}{$\Delta$} & \multirow{3}{*}{$\mathbf{t}^{\star}$} & \multirow{3}{*}{$\mathbf{p}$} \\
\hline & & & & & & & \\
\hline & Mean & SD & Mean & SD & & & \\
\hline Pain in Arm/ Shoulder & 21.1 & 30.9 & 8.8 & 23.0 & $12.3^{\dagger}$ & 2.00 & 0.054 \\
\hline Thoracic pain & 17.7 & 32.4 & 7.7 & 18.9 & $10.0^{\dagger}$ & 1.43 & 0.163 \\
\hline Cough & 51.1 & 31.2 & 42.2 & 31.4 & $8.9^{\dagger}$ & 1.49 & 0.147 \\
\hline Hemoptysis & 8.8 & 23.0 & 0 & 0 & $8.8^{\dagger}$ & 2.11 & $0.043^{\ddagger}$ \\
\hline Dyspnea & 29.6 & 23.2 & 26.3 & 26.0 & 3.3 & 0.66 & 0.510 \\
\hline Dysphagia & 4.4 & 14.4 & 2.2 & 8.4 & 2.2 & 0.70 & 0.489 \\
\hline Stomatitis & 3.3 & 18.2 & 3.3 & 10.1 & 0.0 & 0.00 & 1.000 \\
\hline Pain in other parts & 35.5 & 39.0 & 41.1 & 39.8 & $-5.6^{\dagger}$ & -0.58 & 0.562 \\
\hline Sensory neuropathy & 14.4 & 25.7 & 23.3 & 26.4 & $-8.9^{\dagger}$ & -1.21 & 0.234 \\
\hline Alopecia & 5.5 & 19.7 & 30.0 & 35.39 & $-24.5^{\dagger}$ & -3.95 & $0.000^{\ddagger}$ \\
\hline
\end{tabular}

*Paired t test; +Clinically-relevant scoring; $\neq p<0.05$

In Figure 1, it may be observed that the patients' survival varied from 4 to 20 months with a median of
10.5 months. The mortality rate was $63.3 \%$ with 19 deaths. 


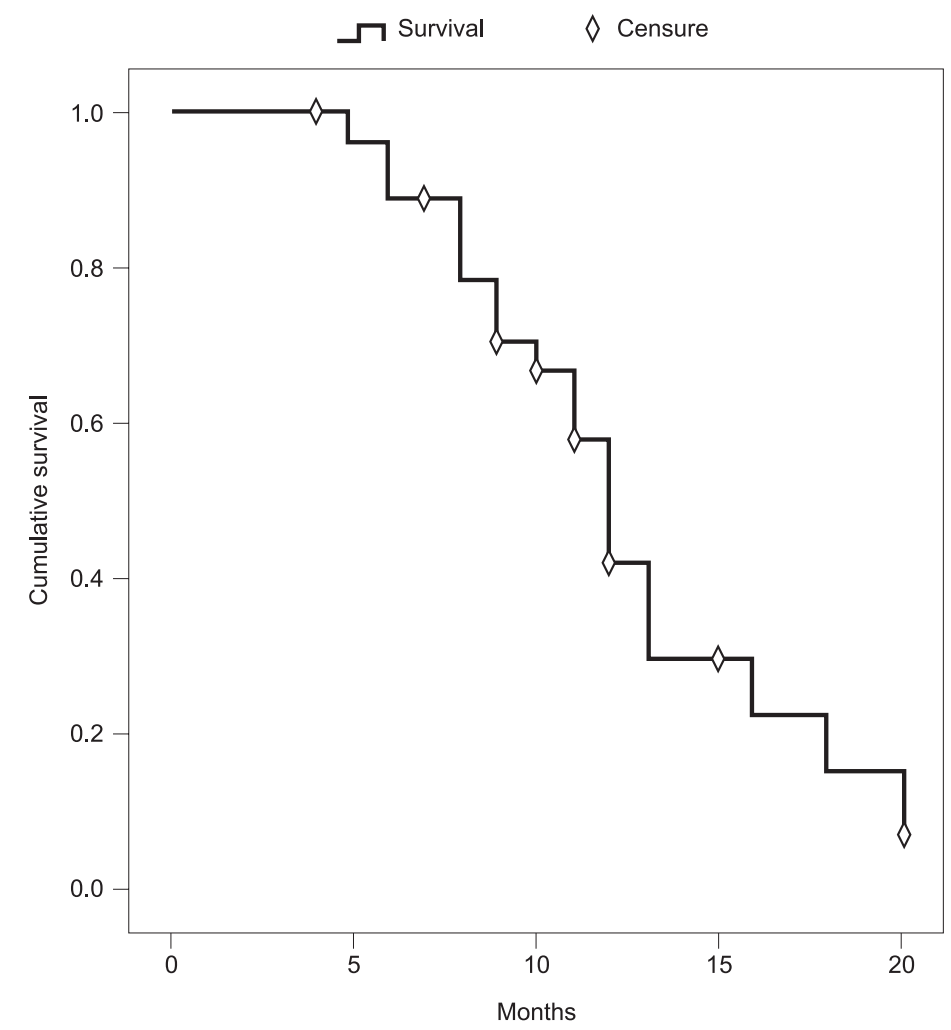

Figure 1 - Survival curve in thirty patients with bronchial carcinoma, São Paulo, SP, Brazil, 2012

\section{Discussion}

In the present study, one can observe a predominance of men with an average age of 68 . The incidence rates for lung cancer hit the age range between 50 and 70 years old and, generally, are higher among men than among women(15).

In the clinical characterization of the sample, the authors observed a large number of patients who smoked or were ex-smokers (83\%). The disease was detected at an advanced stage in $53 \%$ of the patients. In the evaluation of the Karnofsky Scale, there was an average decline of four points over the course of the treatment. Regarding the chemotherapy treatment, 53\% of the patients responded to the treatment, and due to the good Performance Status of this sample's patients, the most-used chemotherapy protocol was Cisplatin + Gemcitabine (63\%). During the study, $63.3 \%$ of the patients died, showing the disease's high lethality. The median survival was 10.5 months.

The treatment of choice for patients in whom the disease is advanced is Adjuvant chemotherapy(7). In the present study, the authors opted for selection of patients with stage IIIB and IV, because these patients are generally prescribed chemotherapy treatment exclusively, as the association of two therapeutic modalities could configure a bias in the assessment of the QLRH related to chemotherapy treatment.

Due to the difficulty in obtaining large samples of this population, various studies have been undertaken with the aim of developing a standard for interpreting the results from QLRH questionnaires ${ }^{(14,16)}$. The interpretation of clinical significance is one of the options for assessing results described in the EORTC's Guidelines, which considers a difference of values from 5 to 10 points to be clinically relevant ${ }^{(13)}$. The present study undertook the assessment of the QLRH through statistical and clinically-relevant significance. The classification made by the EORTC'S QL group was used as a benchmark for the assessment of clinically-relevant significance, and it was considered that a difference of 5 to 10 points has an impact on the patient's clinical response. Considering statistical significance, the authors found an improvement only in the symptoms of dyspnea and hemoptysis, and a worsening for alopecia, while for the assessment of clinical significance, one can observe a higher number of changes in the QLRH. These differences may be related to the size of the sample, due to the disease's high lethality index, and due to the difficulty of detecting changes both over time and among the groups of patients studied(14). 
For patients with lung cancer to maintain or improve their QL is as important as their survival time. In one study carried out with the aim of assessing chemotherapy's impact on patients' quality of life, the assessment of QL was undertaken with the EORTC QLQ C-30 questionnaire, with the lung-cancer-specific module LC-17, and by daily diary cards in two groups of patients: without chemotherapy $(n=138)$ and with chemotherapy $(n=135)$. The study demonstrated there to be no significant differences between the groups, concluding that the chemotherapy does not have a significant impact on the patients' $\mathrm{QL}^{(3)}$.

The findings of the present study demonstrate that the patients' QLRH improved after the chemotherapy treatment. Similar data was found in two studies from outside Brazil which assessed the impact of different chemotherapy regimes on the QLRH of patients with lung cancer. The results indicate that the main chemotherapeutic medications available on the market improve the patients' QLRH and alleviate the symptoms of cancer(17-18)

Dyspnea is one of the three symptoms mentioned most by patients with lung cancer. In the present study, dyspnea was reported by $57 \%$ of the patients at the time of their diagnosis, and after chemotherapy there was an improvement in this symptom. Studies show that dyspnea is a strong predictor of the $\mathrm{QLRH}^{(19-20)}$.

Sleep disturbances are a common problem in cancer patients(21). This sample's patients, however, presented an improvement in the symptom of insomnia after the third cycle of chemotherapy. This higher level of difficulty in sleeping at the start of treatment may be explained by the worry about the disease and anxiety about chemotherapy.

The pain associated with lung cancer may be triggered by various factors, such as the progression of the disease, or may be related to the therapeutic modality(22). In this study the authors observed an improvement in pain in the arm/shoulder and thorax; however, there was a worsening of pain in other parts and peripheral neuropathy after treatment.

Alterations in the gastro-intestinal tract are frequently observed during chemotherapy, and vary according to the medication used. Currently, the chemotherapeutics in clinical use are tolerated well by the patients and have a low toxicity ${ }^{(3)}$. However, the worsening found in this study of the symptoms of fatigue, nausea and vomiting, loss of appetite and alopecia after chemotherapy treatment were also observed in another study carried out, in which the patients who received chemotherapy treatment exclusively has a greater incidence of these symptoms when compared to patients who received radiotherapy exclusively ${ }^{(23)}$.

Currently, the QLRH questionnaires must meet a set of criteria and attributes so as to ensure a reliable measurement ${ }^{(24)}$. It is recommendable that the assessment of internal consistency should be undertaken whenever the instrument is used so as to analyze its behavior in different samples and environments(25). In this study, the instrument for assessing the QLRH demonstrated low rates of reliability in the pain scales, the scale for physical function and nausea and vomiting, in a way similar to what was verified in other studies ${ }^{(10-11)}$

It is very difficult to undertake prospective studies with people with lung cancer, due to the disease's high lethality. A high number of losses may be observed in this sample due to deaths which occurred while chemotherapy treatment was taking place. In terms of clinical relevance, the authors observed important alterations in the patients' QLRH which were not confirmed by the statistical tests, probably due to the sample size.

\section{Conclusion}

In relation to the alterations in the QLRH of the patients with lung cancer after chemotherapy treatment, the authors conclude that there was a clinicallyrelevant improvement in the QLRH, financial difficulties, and in the symptoms of dyspnea, insomnia, cough, hemoptysis, thoracic pain and pain in the arm/shoulder However, there was worsening of the functional scale of role performance and in the symptoms of constipation, alopecia, fatigue, nausea and vomiting, sensory neuropathy, pain in other parts and loss of appetite. The patients had improvement in the QLRH and symptoms related to lung cancer after chemotherapy treatment, however, there was worsening of symptoms resulting from the toxicity of the chemotherapeutic medications. The results obtained in the assessments of QLRH can be an important tool for guiding the health team's interventions in the domains of the QLRH which are most affected according to the therapeutic modality chosen and the patient's clinical progression. Further studies with larger samples must be undertaken to confirm the results obtained in this study.

\section{References}

1. Ministério da Saúde (BR). Câncer no Brasil: dados dos registros de base populacional. Rio de Janeiro (RJ): 
Secretária de Assistência a Saúde; Instituto Nacional do Câncer; 2010; v.4; 463 p.

2. Ministério da Saúde (BR). Estimativa 2012: incidência de câncer no Brasil. Rio de Janeiro (RJ): Instituto Nacional de Câncer; 2011; 118 p.

3. Brown J, Thorpe H, Napp V, Fairlamb DJ, Gower $\mathrm{NH}$, Milroy R, et al. Assessment of quality of life in the supportive care setting of the big lung trial in non-smallcell lung cancer. J Clin Oncol. 2005;23(30):7417-27.

4. Spiro SG, Rudd RM, Souhami RL, Brown J, Fairlamb DJ, Gower NH, et al. Chemotherapy versus supportive care in advanced non-small cell lung cancer: improved survival without detriment to quality of life. Lung Cancer. 2004;59:828-36.

5. Boery EN. Qualidade de vida de pacientes adultos com câncer de pulmão, submetidos à quimioterapia ambulatorial [tese de doutorado]. São Paulo: Escola Paulista de Medicina; 2003. 159 p.

6. Patrick DL, Erikson, P. Health status and health policy. Oxford: Oxford University Press, 1993.

7. Mohan A, Randeep G, Ashutoshk P, Manisha B, Hemraj $P$, Mohan $C$, et al. Quality of life mensures in lung cancer. Indian J Cancer. 2005;42(3):125-32.

8. Karnofsky DA, Burchenal JH. The clinical evaluation of chemotherapeutic agents in cancer. In: McLeod CM ed. Evaluation of chemotherapeutic agents. New York (NY): Columbia University Press. 1949; 191-205 p.

9. Aaronson NK, Ahmedzai S, Bergman B, Bullinger M, Cull A, Duez NJ, et al. The European Organisation for Research and Treatment of Cancer QLQ-C30: A qualityof-life instrument for use in international clinical trials in oncology. J Natl Cancer Inst. 1993; 85(5):365-76.

10. Brabo EP. Brazilian version of the QLQ-LC13 lung cancer module of the European Organization for Research and Treatment of Cancer: preliminary reliability and validity report. Qual Life Res. 2006;15(9):1519-24.

11. Pais-Ribeiro J, Pinto C, Santos C. Validation study of the portuguese version of the QLQ-C30-V3. Psicologia, Saúde \& Doenças. 2008;9(1):89-102.

12. Nunnally JC. Psychometric Theory. New York (NY): McGraw-Hill; 1978. 640p.

13. Young $T$, De Haes $H$, Curran $D$, Fayers $P$, Brandberg $Y$, Vanvoorden $V$, et al. Guidelines for assessing quality of life in EORTC clinical trials. Brussels (BE): EORTC Quality of Life Group; 2002. 32p.

14. Sloan JA, Symonds T, Vargas-Chanes D, Fridley B. Practical guidelines for assessing the clinical significance of health related quality of life changes within clinical trials. Drug info J. 2003;37(1):23-31.
15. Tonani M, Carvalho EC. Cancer risk and preventive behavior: persuasion as an intervention strategy. Rev. Latino-Am. Enfermagem, 2008;16(5):864-70.

16. King MT. The interpretation of scores from the EORTC quality of life questionnaire QLQ-C30. Qual Life Res. 1996;5(6):555-67.

17. Belani CP, Pereir JR, Pawel JV, Pluzanska A, Gorbounova V, Kaukel E, et al. Effect of chemotherapy for advanced non-small cell lung cancer on patients' quality of life a randomized controlled trial. Lung Cancer. 2006;53:231-9.

18. Gebbia V, Lorusso V, Galetta D, Caruso M, Palomba G, Riccardi F, et al. First-line cisplatin with docetaxel or vinorelbine in patients with advanced non-smallcell lung cancer: A quality of life directed phase II randomized trial of Grupo Oncologico Italia Meridionale . Lung Cancer. 2010;69(2):218-24.

19. Joyce M, Schwartz S, Huhmann M. Supportive care in lung cancer. Semin Oncol Nurs. 2008;24(1):57-67.

20. Gupta D, Lis CG, Grutsch JF. The relationship between dyspnea and patient satisfaction with quality of life in advanced cancer. Support Care Cancer. 2007;15(5): 533-8.

21. Chen $\mathrm{ML}$, Yu CT, Yang $\mathrm{CH}$. Sleep disturbances and quality of life in lung cancer patients undergoing chemotherapy. Lung Cancer. 2008;62(3):391-400.

22. Mercadante S, Vitrano V. Pain in patients with lung cancer: Pathophysiology and treatment. Lung Cancer. 2010;68(1):10-15.

23. Piamjariyakul U, Williams PD, Prapakorn S, Kim M, Park L, Rojjanasrirat W, Williams AR. Cancer therapyrelated symptoms and self-care in Thailand. Eur J Oncol Nurs. 2010;14(5):387-94.

24. Camps C, del Pozo N, Blasco A, Blasco P, Sirera R. Importance of quality of life in patients with non-smallcell lung cancer. Clin Lung Cancer. 2009;10(2):83-90.

25. Aaronson N, Burnan A, Lohr KN, Patrick DL, Perrin E. Assessing health status and quality-of-life instruments: attributes and review criteria. Qual Life Res. 2002;11(3):193-95. 